Service social

\title{
La Loi sur la protection de la jeunesse et les travailleurs sociaux : impacts sur leurs valeurs, sur leur pratique et sur leur formation
}

\section{Pierre Pinard}

Volume 40, numéro 2, 1991

Formation et évolution de la pratique en travail social

URI : https://id.erudit.org/iderudit/706525ar

DOI : https://doi.org/10.7202/706525ar

Aller au sommaire du numéro

Éditeur(s)

École de service social de l'Université Laval

ISSN

1708-1734 (numérique)

Découvrir la revue

Citer cet article

Pinard, P. (1991). La Loi sur la protection de la jeunesse et les travailleurs sociaux : impacts sur leurs valeurs, sur leur pratique et sur leur formation. Service social, 40(2), 26-42. https://doi.org/10.7202/706525ar

\section{Résumé de l'article}

La Loi sur la protection de la jeunesse a considérablement heurté les valeurs et les attitudes des travailleurs sociaux, elle a modifié l'objet de leur pratique et elle a entraîné une gestion des services sociaux en protection de la jeunesse qui se traduit par une forte segmentation de la pratique sociale. Face à ces changements profonds, les travailleurs sociaux en sont arrivés au fil des années à un degré d'adaptation et d'acceptation qui surprend.

Ces modifications entraînent cependant des ajustements à leur formation. Ils devront en effet posséder un solide cadre de référence, une bonne identité professionnelle et une bonne maîtrise du processus clinique. Il leur faudra être adaptables, polyvalents et surtout capables de partager avec d'autres un processus clinique dans lequel ils interviendront partiellement. 


\section{La Loi sur la protection de la jeunesse et les travailleurs sociaux : impacts sur leurs va- leurs, sur leur pratique et sur leur formation}

Pierre Pinard

\section{Introduction}

L'Assemblée nationale a adopté à l'unanimité la Loi sur la protection de la jeunesse en 1977. Ce faisant, le Québec amorçait un virage et manifestait une volonté de société dont les effets sont encore mal saisis. Dans son essence, cette nouvelle loi affirmait quatre principes qui constituent des orientations pour la pratique sociale en protection :

1. Les mesures d'ordre social et volontaires doivent primer sur les mesures judiciaires (la déjudiciarisation).

2. L'enfant, d'objet de droit qu'il était, devient sujet de droit.

3. La loi fait obligation aux intervenants de favoriser le maintien de I'enfant dans son milieu naturel.

4. La responsabilité de l'enfant incombe en premier lieu à ses parents.

Depuis l'entrée en vigueur de cette loi en janvier 1979, plusieurs spécialistes du domaine, professeurs, intervenants ou cadres, en ont fait l'analyse. On y retrace une évolution des attitudes des travailleurs sociaux qui part de la résistance, passe par l'apprivoisement et finit par l'accommodation sur le plan des valeurs.

La Loi sur la protection de la jeunesse a considérablement heurté les valeurs et les attitudes des travailleurs sociaux, a modifié l'objet de leur pratique et elle a entraîné une gestion des services sociaux en protection de la jeunesse qui se traduit par une forte segmentation de 
la pratique sociale. Face à ces changements profonds, les travailleurs sociaux en sont arrivés au fil des années à un degré d'adaptation et d'acceptation qui surprend.

Ces modifications entraînent cependant des ajustements à leur formation. Ils devront en effet posséder un solide cadre de référence, une bonne identité professionnelle et une bonne maîtrise du processus clinique. II leur faudra être adaptables, polyvalents et surtout capables de partager avec d'autres un processus clinique dans lequel ils interviendront partiellement.

\section{Impacts sur les valeurs des travailleurs sociaux}

\section{Valeurs remises en cause}

Les travailleurs sociaux n'ont pas mis beaucoup de temps à saisir que la Loi sur la protection de la jeunesse remettait en question certaines de leurs valeurs les plus chères. Et cette réalité, Pierre Racine ${ }^{1}$ l'a admirablement bien décrite en analysant la situation d'un CSS en 1984 :

Nous postulons que les rationalités professionnelle et technocratique ne sont pas "en soi » incompatibles.

Les intervenants vivent toujours une contradiction profonde entre une idéologie humaniste de l'aide qui les traverse et la fonction de " contrôle social » qui est inévitablement liée à l'activité de socialisation dans laquelle leur pratique les place. Cette contradiction, non résolue, les amène à vivre leur pratique comme des " agents doubles " tout en gardant la nostalgie d'agir comme des " agents libres" avec des clients " motivés " et " volontaires ". Ils sont d'accord, par exemple, avec la mission protection, mais souhaiteraient pouvoir la remplir sans avoir à recourir au contrôle ou en faisant accomplir cette fonction par d'autres.

La représentation sociale de base de l'intervention à laquelle la pratique professionnelle se réfère comporte des contradictions et des lacunes importantes que la rationalité technocratique ne permet plus de garder à l'état latent. La vision de société qui découle de cette représentation est celle d'une société sans conflits où les rapports sociaux semblent tenir une place plus mythique que réelle dans la mesure où leurs conséquences concrètes ne sont pas reconnues dans les situations traitées.

Dans le cadre des travaux de la Commission Rochon, Blanchet et Laflamme ${ }^{2}$ énonçaient un point de vue assez semblable. Ils notaient une certaine morosité chez les travailleurs sociaux principalement due au débat portant sur la relation d'aide et la relation d'autorité, à l'aug- 
mentation de la bureaucratie, à la diminution du temps d'exposition à la clientèle.

Bon nombre d'intervenants se sont bien adaptés à ce contexte plus cadré. Les autres ont massivement opté pour les CLSC dans le cadre de partage CSS-CLSC.

\section{Vers l'apprivoisement et l'intégration de nouvelles valeurs}

À compter de 1984, tout en reconnaissant que la Loi sur la protection de la jeunesse entraîne des modifications profondes de valeurs, d'attitudes et de pratiques, certains spécialistes commencent à accepter la fonction de contrôle social imposée par cette loi, et y voient des retombées positives. C'est notamment le cas de trois intervenants en protection de la jeunesse du CSSMM énonçant un credo qui n'est pas démuni de sens ${ }^{3}$.

De multiples " rationnels " ont été élaborés par les tenants du noninterventionnisme et par ceux qui remettent en question les organismes technocratiques et bureaucratiques. Il serait imprudent de faire fi de ces analyses, d'écarter aveuglément toute critique et de fermer les yeux sur une portion importante du réel. Mais nous pensons que notre rôle comporte une dimension de responsabilité sociale et que personne, ni aucune de ces approches, ne peut décemment cautionner le laisser-faire au nom de la liberté ou de quelque autre valeur, lorsqu'il s'agit de la sécurité d'enfants inaptes à assurer eux-mêmes les conditions minimales de leur développement ou de leur sécurité. Et c'est dans cette perspective qu'il nous faut admettre :

- que le droit à l'autodétermination n'est pas un droit absolu;

- que l'acceptation inconditionnelle, le respect et le non-jugement, longtemps valorisés en service social, ne signifient pas et n'ont jamais signifié qu'il faille tolérer l'abus ou des agirs inadéquats; on peut fort bien reconnaître la valeur et la dignité de la personne humaine sans entériner des comportements, par ailleurs inacceptables, à l'égard de la dignité et de la valeur de l'autre personne qu'est l'enfant.

II semble donc que, dans la pratique, on puisse concilier un mandat de protection avec les valeurs traditionnelles de la discipline du travail social. II apparaît donc possible de faire du travail social en protection de la jeunesse.

\section{Un cadre de référence encore essentiel}

Au Québec, depuis le milieu des années trente, les travailleurs sociaux œuvrent auprès des pauvres, des démunis et des familles en difficulté. La discipline du travail social traduit une vision spécifique de 
I'humain : les comportements et les attitudes sont la résultante des facteurs socio-économiques et des facteurs de personnalité. Nous savons que la pauvreté est intimement associée à la scolarisation, au revenu et au statut et qu'en protection de la jeunesse, nous faisons constamment face à toutes les formes de pauvreté.

Ainsi, lorsque le travailleur social statue sur la sécurité ou le développement d'un enfant, il le fait en relativant (contextant) le geste parental dans un environnement social donné.

Le cadre de référence est et doit absolument être réaffirmé et intégré. C'est lui qui permet au travailleur social de garder son identité et sa raison d'être lorsque sa fonction l'amène à intervenir dans un domaine de contrôle social; c'est lui qui lui permet de concilier aide, surveillance et contrôle.

\section{Changement dans les valeurs de fond}

Les travailleurs sociaux et les autres professionnels évoluent dans et avec la société, une société dans laquelle on observe de plus en plus d'intolérance. Ils sont plus stressés, plus agressifs, plus défensifs à l'endroit de clients plus exigeants, mieux informés et soutenus (apport du monde juridique).

Quoique interdépendants, ces professionnels ont de la difficulté à élaborer des projets collectifs (travail en réseau), ils sont individualistes et s'accommodent bien d'une tâche précise, bien ciblée, dont ils ont la maîtrise et qui leur procure une satisfaction. Bref, dans une ère de haute technologie, ils avancent avec la vague.

\section{Impacts sur la pratique sociale}

Si la Loi sur la protection de la jeunesse (LPJ) a suscité des accommodations de valeurs et d'attitudes des travailleurs sociaux, elle a aussi bouleversé considérablement la pratique du travail social. Ce bouleversement n'est certes pas étranger aux réactions que la LPJ a suscitées chez les travailleurs sociaux dès 1979, et qu'elle continue de faire naître dans le réseau.

\section{Modifications dans la finalité de la pratique sociale}

La pratique d'une discipline c'est l'application, l'exécution, la mise en action d'une théorie. La pratique appelle donc un encadrement théorique d'ordre cognitif et méthodologique déterminé par des buts et des objectifs à poursuivre ${ }^{4}$. 
Notre prétention est que la LPJ a modifié en partie la finalité de la pratique du travail social en limitant sa portée. La discipline du travail social véhicule une vision spécifique de l'humain et vise à restaurer le fonctionnement social des personnes, des familles et des groupes de façon satisfaisante pour eux et leur milieu. Cet objectif de l'acte professionnel du travailleur social peut être atteint, dans le cadre de la collaboration de l'ensemble des organismes assujettis à la Loi sur les services de santé et les services sociaux, mais devient impossible à réaliser dans sa totalité dans le seul cadre de la LPJ.

La LPJ, en contrepartie de l'autorité qu'elle confère, limite et cible une finalité de l'intervention qui n'est pas un objectif global en soi. En protection de la jeunesse, l'intervention doit cesser dès que la sécurité et le développement (compris dans le sens de réponses aux besoins essentiels) de l'enfant ne sont plus compromis et dès que le risque de récurrence est minimalement jugulé.

Ce sont les limites accolées aux concepts de sécurité et de développement compromis qui sont au cœur des débats et des frustrations des intervenants du réseau depuis l'entrée en vigueur de la LPJ. Plus que les moyens (interventions qui ne parviennent pas toujours à corriger la situation), plus que la segmentation du travail, plus que les impératifs du système judiciaire, c'est le fait de réduire à des conditions minimales les critères de sécurité et de développement de l'enfant qui fait surgir la résistance, voire la révolte chez les travailleurs sociaux.

Un an à peine après I'application de la LPJ, l'étude de Lesemann et Renaud ${ }^{5}$ captait ces transformations profondes de la pratique :

Il s'agit d'une recherche que nous avons eu l'occasion d'effectuer récemment dans un CSS et qui portait sur les effets de la mise en application de la loi 24 , sur les pratiques professionnelles dans les services de prise en charge (services Famille-Enfance) des cas confiés par la direction de la protection de la jeunesse... L'implantation de la DPJ entraîne donc une transformation profonde de la pratique mais aussi des finalités de cette pratique.

À notre point de vue, c'est davantage par la segmentation de la finalité de la pratique que l'État modifie la pratique sociale. II renferme le travailleur social dans des espaces restreints : services sociaux courants, services sociaux de deuxième ligne, protection de la jeunesse. Une nouvelle conception de la pratique sociale est imposée de l'extérieur. Ce nouveau modèle de pratique contraint le travailleur social qui recherche le complément de son acte professionnel à l'acceptation profonde d'une forme de travail à relais. La logique du travail à relais repose sur la nécessaire complémentarité et compétence de celui qui est en amont et de celui qui est en aval du relais. En d'autres mots, l'acte du travailleur social n'est complet dans sa finalité que dans la mesure 
où il est complété par un ou plusieurs autres intervenants. Et pour des individualistes, cela devient très irritant.

\section{Concentration en protection de la jeunesse d'une clientèle en sévère difficulté}

Cette conception de la pratique a pour effet immédiat de concentrer en protection de la jeunesse uniquement les situations graves et dramatiques.

Quelle que soit la nature de la maltraitance en cause, les situations de protection présentent des caractéristiques générales constantes. Ce sont toujours des situations de crise, des situations indicatrices de problèmes, révélatrices, mouvantes, complexes et chroniques ${ }^{6}$.

Très souvent, les familles qui font l'objet de la protection de la jeunesse vivent des problèmes sur plusieurs plans à la fois : physiques, psychologiques, économiques, sociaux, relationnels. Ces familles se caractérisent par une instabilité émotionnelle, éducative et de milieu physique. Les cas de négligence, notamment, qui sont les plus nombreux, proviennent le plus souvent de sous-cultures ou de milieux marginaux. Enfin, une dernière caractéristique des situations de protection réside dans la présence de violence à un degré élevé et selon différents registres.

Outre les exigences de la clientèle, il faut aussi être conscient que le milieu exerce une pression inouïe sur les travailleurs sociaux en protection de la jeunesse. Il s'attend à ce qu'ils résolvent des problèmes éminemment complexes en étant constamment surveillés par les médias en quête de sensationnalisme, par la Commission de protection des droits des enfants qui, elle, ne tient pas compte des limites du système de protection et par les signalants qui trouvent que les travailleurs sociaux font trop peu et trop tard.

Il est tout à fait probable que les travailleurs sociaux aient peu ou pas réagi à la très grande segmentation dans la livraison des services sociaux parce que cette division des tâches intervient comme un allègement d'une réalité d'intervention qui serait autrement écrasante. II s'agirait donc en quelque sorte d'une réaction de survie.

\section{Une gestion qui segmente la livraison des services sociaux}

Premier niveau de segmentation : la Loi sur la protection de la jeunesse comme dernière pièce $d u$ puzzle législatif et social

L'obligation de traduire judiciairement des situations familiales et sociales perçues, évaluées et traitées en termes psychosociaux impose 
des contraintes et des limites aux travailleurs sociaux. Les règles de procédures judiciaires aussi. Au-delà d'une nécessaire complicité socio-judiciaire accrue, les fondements et les limites de la LPJ ne sont pas remis en cause par les travailleurs sociaux.

Dans la mesure où l'on saisit correctement la complémentarité de nos législations, la Loi sur la protection de la jeunesse constitue un cadre législatif suffisamment large et généreux pour assurer la protection effective des enfants québécois, sans pour autant influer d'une manière indue sur la responsabilité première des parents en ce qui a trait à l'éducation des enfants. En effet, le Code civil, qui s'applique à l'ensemble de la population, affirme la présomption de la capacité parentale. La Loi sur les services de santé et sur les services sociaux rend accessibles des soins et des services sur base volontaire aux personnes et familles qui éprouvent des difficultés à s'acquitter de leurs responsabilités parentales ou autres. La LPJ, quant à elle, garantit, même de façon coercitive, la sécurité et le développement minimal de chaque enfant. La logique législative va du général au très particulier.

Protéger les enfants québécois suppose une concertation interréseaux des affaires sociales. Or, à ce chapitre, les acquis ne sont pas suffisants pour constituer des gages certains de réussite et de performance.

L'application de la protection de la jeunesse continue d'être la responsabilité à peu près exclusive des CSS, en dépit des protocoles existant avec plusieurs établissements du réseau.

Bref, notre point de vue est le suivant :

Nous vivons dans une société qui se dote de politiques, de lois, de programmes et de services, qui mandate des ministères, des établissements, des centres pour gérer tout cela. Chacun en fonction d'une mission donnée. Ce qui frappe au premier chef, c'est la nécessaire interdépendance et intercomplémentarité de ces acteurs sociaux.

L'harmonisation du réseau n'étant pas faite, il se trouve des enfants et des familles en détresse sociale qui ne sont reçus ni à la protection de la jeunesse, ni dans les CLSC, ni ailleurs. Ces cas demeurent sans réponse.

Cette conception segmentée de livraison des services sociaux peut être remise en question tant sur le fond que dans son applicabilité. Répartir les services jeunesse-famille sur deux catégories d'établissements, les CLSC et les CSS, en fonction de missions qui demeurent floues (cadre de partage CSS-CLSC de 1984) crée une brisure dans le continuum des besoins des clientèles et dans la pratique sociale. C'est un choix sur lequel il faut s'interroger. 
Quant à l'applicabilité de ce choix, force est de reconnaître que les efforts déployés depuis 1984 pour harmoniser les missions des deux catégories d'établissements se révèlent sans résultats probants. Amener différents établissements à contribuer à des résultats communs constitue un défi colossal.

Deuxième niveau de segmentation :

division des tâches et spécialisation dans les directions de protection de la jeunesse (le rapport Harvey comme principal héraut)

\section{- Resserrement du mandat P.J.}

De l'aveu même des DPJ de la première heure et qui sont encore en place, quand ils ont mis sur pied le système de protection de la jeunesse en 1979, ils en " ramassaient » trop large. À ce moment-là, la Loi sur la protection de la jeunesse englobait aussi les jeunes délinquants et le concept même de protection était perçu dans une extension large.

L'absence de ressources, la très grande visibilité sociale de la protection de la jeunesse, de même que sa remise en question constante, a forcé les gestionnaires et les intervenants en protection de la jeunesse à redéfinir, circonscrire, limiter, préciser.

Dans une analyse comparant le concept de protection québécois et le concept ontarien en 1987, Blanchet et Pinard ${ }^{7}$ constatent que les choses s'améliorent. Ils reconnaissent que le concept de protection est mieux perçu et balisé, que les CSS ont développé une expertise enviable en protection de la jeunesse et que les nouveaux intervenants disposent d'une méthodologie rassurante et efficace.

Par ailleurs, même si le concept se fait de plus en plus précis et limitatif, ils énoncent que les concepts de protection sociale, de développement mental et affectif, de troubles sérieux de comportements gagneraient à être clarifiés.

Ils déplorent de plus que les autres organismes et notamment les CLSC ne contribuent pas assez à la protection de la jeunesse. II y a l'absence de volonté politique de le faire, mais il y a aussi la difficulté de cerner quand finit la P.J. et quand débute le besoin d'aide.

Ils poursuivent en affirmant que :

Depuis 1979, les CSS ont développé malgré les contraintes une expertise enviable en protection : précision des signalements, évaluation de situations complexes en contexte non volontaire, traitement des abus sexuels, protocoles d'intervention, protocles socio-juridiques, programmes de formation, utilisation et collaboration avec les ressources du milieu. Les intervenants disposent maintenant d'une mé- 
thodologie rassurante et efficace dans certains secteurs d'activités. II faut maintenant poursuivre, expérimenter de nouvelles avenues et évaluer les résultats de façon scientifique.

Les intervenants demandent la création de conditions professionnelles et de travail telles qu'elles attirent et retiennent les meilleurs éléments. Or, les conclusions qui se dégagent des consultations de groupes de professionnels vont dans un sens diamétralement opposé : le contexte de la pratique et l'absence de support professionnel rendent difficiles des interventions de qualité.

\section{- Mandat du groupe Harvey}

C'est aussi en 1987 que fut décidée la création d'un groupe de travail sur l'application de la Loi sur la protection de la jeunessse.

Avec l'assentiment de l'Association des Centres de services sociaux, le ministère de la Santé et des Services sociaux créait un groupe de travail sur l'application de la protection de la jeunesse dans les CSS (Rapport Harvey, phase I) ${ }^{8}$.

Le groupe de travail inter-CSS fut mandaté par le ministère de la Santé et des Services sociaux du Québec pour procéder à une analyse des activités de réception et de traitement des signalements (RTS), et d'évaluation et d'orientation (EO) en protection de la jeunesse (PJ). Les objectifs de cette démarche étaient de dégager une image plus précise des processus d'intervention et des modes de fonctionnement dans différents CSS, de manière à identifier des avenues d'amélioration du processus.

En avril 1988, la Ministre de la Santé et des Services sociaux acceptait le rapport sur l'analyse des activités de réception et de traitement des signalements, d'évaluation et d'orientation en protection de la jeunesse (Rapport Harvey), et demandait que les recommandations de ce rapport basé sur les travaux du groupe de travail inter-CSS et MSSS soient implantées dans tous les CSS $^{9}$.

Sans diminuer l'importance et l'impact du rapport Harvey, son principal mérite a été d'ordonner, d'uniformiser la pratique à travers les 14 entités particulières que sont les CSS, à partir d'un modèle largement répandu. Ce savoir-faire, il l'a enchâssé dans des principes de division et de spécialisation du travail.

\section{- Principes présidant à la division et à la spécialisation du travail en protection de la jeunesse}

Le processus de protection de la jeunesse est unique en son genre. Nulle part ailleurs rencontre-t-on des situations où des groupes de professionnels " égaux » doivent travailler, somme toute à la chaîne, avec l'uniformité et la cohérence requise.

Le processus de protection de la jeunesse, tel qu'il s'applique actuellement dans la majorité des CSS, consiste en un découpage en trois 
étapes discrètes d'un processus continu. Les trois étapes sont découpées séquentiellement de la plus courte à la plus longue.

II faut donc chercher à atteindre et à maintenir un équilibre dynamique, à l'intérieur duquel chaque étape du processus utilise au maximum son pouvoir de discrimination, sans pour autant prendre de risque excessif et sans chercher à se substituer à l'étape subséquente $^{10}$.

Ainsi donc, tel que défini par Harvey l, le processus en protection de la jeunesse se réalise en quatre étapes : réception et traitement des signalements, évaluation, orientation, auxquels s'ajoute une quatrième étape, le processus d'application des mesures. Il est assuré par trois groupes de professionnels affectés à des mandats distincts mais égaux, qui se relayent les clients les uns les autres, tout en maintenant un même esprit et en poursuivant les objectifs de la LPJ.

De la même façon que nous avons remis en question la segmentation de la pratique sur plusieurs établissements, il convient aussi de nous interroger sur le travail à relais à l'intérieur des pratiques en CSS. L'organisation du travail et des pratiques suppose un partage parfait des concepts, des programmes, des critères d'action entre les intervenants et entre les services. Or, on observe souvent qu'il existe entre les intervenants et entre les services des suspicions, voire des rivalités. En conséquence, il faut éviter une trop grande division du travail. II conviendrait même de garder uni sous un même service et sous un même intervenant tout ce qui est conciliable. II y a donc des choses à revoir, tant sur le plan de la gestion que sur le plan des attitudes (savoirêtre) des intervenants.

\section{La spécialisation en protection de la jeunesse}

Plus les exigences sont fortes quant à l'atteinte d'une cible précise et limitée, plus le champ d'intervention doit être restreint et plus l'équipement doit être perfectionné. C'est la logique qui préside à la division du travail et à la spécialisation en protection de la jeunesse.

On a constitué des équipes centralisées de RTS pour restreindre le nombre de personnes ayant une emprise sur l'entrée des cas. II est ainsi plus aisé de gérer l'extension du concept de protection à l'entrée. Et la décision qui résulte du travail du professionnel RTS se limite à décider si le signalement peut être retenu ou pas.

L'équipe Évaluation-orientation (E-O) prend le relais et statue sur la sécurité et le développement compromis ou non et sur le choix de mesures et de régime. Après quoi, elle se retire.

Les équipes RTS et E-O sont obligatoirement sous la responsabilité du DPJ. Parce que les directions de protection de la jeunesse dans les 
CSS ont été favorisées sur le plan budgétaire et parce que leurs professionnels interviennent en fonction de mandats courts, précis et limités, pour lesquels ils ont été formés, le personnel de ces équipes a bénéficié d'une reconnaissance et d'une valorisation de statut professionnel. II en fut et en est encore tout autrement des équipes de prise en charge et notamment dans les CSS où la prise en charge n'est pas sous la responsabilité du DPJ.

Mais la spécialisation ne s'est pas limitée aux trois étapes distinctes. Le raffinement de mandat s'est poursuivi à l'intérieur même de I'E-O. Là où le volume le justifiait, des professionnels E-O se sont concentrés sur des problématiques particulières - abus sexuels,

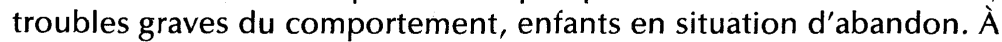
l'exception des programmes de traitement des abus sexuels qui, dans certains CSS, sont remis en question, la spécialisation même à l'intérieur d'un mandat déjà restreint $(E-O)$ est évaluée positivement par les cadres et par les professionnels.

Comme le mentionnait avec justesse Pierre Racine ${ }^{11}$, le rapport Harvey contribue à structurer l'intervention, ce qui représente un acquis sur le plan de l'expertise professionnelle.

Somme toute, la spécialisation par problématique confère de grands avantages et une valorisation certaine aux intervenants et aux cadres qui y gagnent une reconnaissance et une notoriété professionnelle. C'est un des constats auxquels en venait Hélène Manseau dans son analyse du traitement de l'abus sexuel au Québec ${ }^{12}$. Cependant, la spécialisation comporte le risque que la réalité globale soit réduite au seul prisme du spécialiste.

Cette notoriété professionnelle est maintenant revendiquée par les professionnels qui travaillent à l'application des mesures (prise en charge). Ces professionnels n'acceptent plus d'être perçus comme le déversoir de tout ce que les autres ne veulent pas. Ils n'acceptent plus les caseloads hétérogènes où se côtoient négligence, troubles du comportement, abus sexuels, abus physiques, etc.

Ces professionnels revendiquent à leur tour une spécialisation par problématique qui prendrait la forme d'une majeure et d'une mineure dans un caseload donné. Ils insistent pour que la programmation des services se fasse par problématique, en développant en priorité les programmes négligence et troubles du comportement. Ils demandent une meilleure formation et un meilleur encadrement, ils exigent des conditions de pratique qui permettent un travail plus valorisant.

En résumé, ils effectuent aussi le virage de la spécialisation par problématique, de la réduction de la largeur des cibles d'intervention au profit d'une intervention plus documentée, plus précise et dont les résultats sont plus apparents. 


\section{Standardisation}

Que le ministère de la Santé et des Services sociaux et les Centres de services sociaux jugent important de pouvoir mesurer et comparer les activités semblables qu'ils exécutent nous apparaît tenir d'une logique de gestion et d'imputabilité de bon aloi. Harvey propose une division du travail (méthodologie) qui est perçue comme aidante pour les travailleurs sociaux. L'essence même du rapport Harvey porte sur le resserrement de la méthodologie de travail en protection de la jeunesse.

\section{Critères de résultats}

Même si les travailleurs sociaux se réjouissent du fait que les enfants soient protégés plus rapidement, ils ne considèrent pas pour autant que les délais de Harvey I soient les seules et les meilleures mesures de l'efficacité et de l'efficience. La distribution des services sociaux relève d'une logique économique dans laquelle les indicateurs d'efficience sont d'ordre quantitatif : liste d'attente, respect des délais, roulement des dossiers, taux de judiciarisation, rationalisation des effectifs, normes de charges de cas axées sur la quantité sans égard à l'intensité de l'intervention.

II serait sans doute approprié de définir les critères d'efficience en termes de sécurité-développement non compromis (S.D.N.C.), nonrécurrence de la situation, résultats atteints en moins de temps, utilisation parcimonieuse des ressources, réduction du nombre des placements, réinsertion hâtive, etc.

\section{Impacts sur la formation des travailleurs sociaux}

De l'étude des impacts de la LPJ sur les valeurs et la pratique des travailleurs sociaux et de l'observation plus générale de la pratique des intervenants sociaux se dégagent des exigences pour leur formation.

\section{Besoin d'un cadre de référence solide}

Pour intervenir correctement dans le cadre des différents mandats qui leur sont dévolus, il est essentiel.que les travailleurs sociaux puissent situer leurs interventions dans un cadre d'analyse large et complet. Ils doivent bien connaître les pôles société-individu, de même que les interactions entre les deux.

II leur faut connaître comment sont engendrés les phénomènes et les problèmes sociaux, quels en sont les déterminants et comment on 
peut agir sur eux. C'est ainsi qu'ils seront amenés à saisir les déterminants de la santé et du bien-être, à posséder des notions comme celles de rapports sociaux, acteurs sociaux, pouvoir. Ils doivent pouvoir comprendre et expliquer en quoi les conduites humaines sont les résultantes des dynamiques sociétales et personnelles.

Il convient donc qu'ils connaissent bien le développement humain dans ses principales phases. Étant donné qu'un bon nombre d'entre eux seront appelés à exécuter des mandats d'aide, ils doivent être initiés aux principaux problèmes auxquels fait face notre génération : difficulté d'être parent, divorce, monoparentalité, reconstitution familiale, violence conjugale et familiale, maladies mentales, dépendances diverses, pour n'en nommer que quelques-uns.

\section{Besoin d'identité professionnelle}

Au moment où les CLSC voient élargir leur mission, au moment où les centres de réadaptation font le virage milieu et le virage famille ${ }^{13}$, alors que les travailleurs sociaux doivent partager avec plusieurs autres professionnels des processus d'aide, une identité professionnelle forte s'avère essentielle. Quels sont les caractères communs des disciplines d'aide? Quelles sont leurs caractéristiques propres ? Quel est, par exemple, l'apport spécifique du travailleur social lorsqu'il collabore avec un psycho-éducateur à la réinsertion sociale d'un adolescent placé en centre de réadaptation?

Dans cette veine, il serait intéressant que les futurs travailleurs sociaux soient exposés aux pratiques des autres disciplines connexes, que cette exposition se fasse sur le terrain de la pratique.

\section{Adaptabilité et polyvalence}

Les travailleurs sociaux, en cours de carrière, auront à intervenir dans différents milieux, différents établissements et différents programmes. En conséquence, ils doivent pouvoir réaliser rapidement des transferts de connaissances et d'habiletés d'un mandat à un autre. Ils devront notamment ajuster leur art à des mandats qui incluent à la fois des dimensions d'aide, de surveillance et de contrôle. Le fondement de cette capacité de transfert réside dans une formation générale et polyvalente plutôt que dans une formation technique et axée sur une problématique.

\section{Des travailleurs sociaux " interactifs"}

La segmentation des mandats et des pratiques rend impérieuses la capacité et la disposition du travailleur social à composer avec d'autres 
travailleurs sociaux ou d'autres intervenants. Leur apprentissage devra les amener à effectuer des processus cliniques comportant des relais. Ils auront donc à développer la confiance aux autres et leurs capacités de réaliser à plusieurs un mandat commun. Cela exige par conséquent la capacité de se situer et de respecter l'autre, le développement du sens de la responsabilité individuelle mais complémentaire dans un projet commun. Les travailleurs sociaux interactifs devront, de toute évidence, posséder un sens poussé de l'éthique dans leurs rapports avec leurs collègues et avec la hiérarchie.

\section{Maîtrise de la méthodologie du travail social}

On ne saurait trop insister sur l'importance de la maîtrise des différentes étapes du processus clinique : collecte de données, analyse, évaluation, formulation d'objectifs et de moyens, actualisation, évaluation des résultats.

Quoique nécessaire dans tous les domaines, cette habileté devient impérative dans le cadre du mandat de la PJ. Lorsque l'intérêt, la sécurité ou le développement d'un enfant sont en jeu, quand le retrait d'un enfant de son milieu en dépend, l'évaluation de la situation faite par le travailleur social se doit d'être méthodique et fiable.

\section{Connaissance de la législation}

Sans prétendre à une connaissance approfondie des lois canadiennes et québécoises, les travailleurs sociaux doivent posséder une connaissance suffisante des lois qui ont une portée sociale : les différentes chartes, la Loi sur la santé et les services sociaux, la Loi sur la protection de la jeunesse, la Loi sur les jeunes contrevenants, la Loi sur le curateur public, la Loi sur la protection de la santé publique, le Code civil du BasCanada et le Code civil du Québec.

On devrait surtout insister sur la complémentarité de ces pièces législatives, sur la logique du droit, sur la présomption d'innocence, sur les règles de procédure en matière criminelle et au Tribunal de la jeunesse.

\section{Conclusions}

\section{Conclusion quant aux valeurs}

Il est indéniable que les travailleurs sociaux ont résolu le dilemme aide-surveillance et contrôle. La plupart des travailleurs sociaux en protection de la jeunesse sont maintenant à l'aise avec cette fonction 
de contrôle social. Cette aisance, ils la doivent d'abord à un cadre de référence disciplinaire qui permet la relativité de la responsabilité parentale sur l'incapacité parentale. Sans oublier ni renier le mandat de contrôle social, le travailleur social exécute ce mandat en adoptant un esprit de non-culpabilisation des parents. Force est de constater aussi que les travailleurs sociaux ont fait une accommodation que je qualifie $d^{\prime}$ intelligente de leurs valeurs traditionnelles du travail social. L'autodétermination d'un parent qui compromet la sécurité ou le développement de son enfant ne peut être maintenue à l'état pur.

Parents et enfants sont maintenant égaux en valeur et en droit. Le plus faible a droit à l'assistance d'un tiers (le DPJ) lorsque sa sécurité ou son développement sont compromis. C'est un choix de société auquel les travailleurs sociaux s'associent.

\section{Conclusion quant aux changements dans la pratique sociale}

Sans vouloir revenir à 1970, l'époque des agences de services sociaux, dans lesquelles I'accueil, l'évaluation et la prise en charge étaient souvent le fait du même intervenant et du même service, le choix de limiter l'intervention des travailleurs sociaux à l'assurance de sécurité-développement non compromis pose des problèmes. Cette disposition de la LPJ est d'autant plus heurtante pour les travailleurs sociaux qu'ils savent fort bien que le relais pour la poursuite des besoins de services n'est pas assuré. Les travailleurs sociaux ne peuvent accepter que certaines clientèles aient droit et accès à des services qui soutiennent leur développement optimal (les handicapés) et que d'autres (les enfants en besoin de protection) aient droit et accès à des services qui assurent leur sécurité et leur développement dans leurs éléments essentiels seulement et encore avec certains retards. Nous postulons que les soins et services essentiels doivent être accessibles à toute personne sans délai. Les choix de société au-delà de ce plancher minimal doivent être revus dans le sens d'une plus grande équité.

La restriction du mandat des établissements et la limitation des fonctions des intervenants à l'intérieur du même établissement reposent sur certains impératifs de gestion incontournables. Cependant, ce principe de la division du travail doit être relativisé par l'analyse des effets pervers de son application.

Une trop grande multiplicité des relais dans l'actualisation d'un mandat ou d'un processus entraîne souvent beaucoup d'incohérence, de confusion de rôles et même la perte pure et simple de l'objectif du mandat ou de l'intervention. 


\section{Conclusion quant à la formation des travailleurs sociaux}

Sans proposer un programme détaillé des exigences de la formation des travailleurs sociaux, nous insistons particulièrement sur la nécessité d'un cadre de référence, d'une bonne identité professionnelle, d'une capacité d'adaptation à différents mandats et sur une connaissance des lois sociales.

Nous insistons davantage encore sur le savoir-faire (maîtrise de la méthodologie du travail social). Et parce que cette dimension s'avère plus difficile à acquérir, bien qu'elle soit essentielle, le savoir-être (particulièrement à l'endroit des collègues) mérite toute notre attention. Sans cette capacité d'interaction et de complémentarité des différents intervenants, le réseau public risque de s'avérer inefficient, voire inopérant.

À cette formation de base dispensée par les universités doit se greffer une formation continue. Chaque fois qu'un travailleur social change de mandat ou de programme, chaque fois que l'État ou l'employeur introduit une modification d'orientation ou de pratique, ces modifications dans l'exercice d'une discipline devraient être appuyées par une formation d'appoint. Et à ce chapitre, selon la Commission Rochon, nous accusons un retard important.

\section{Perspective globale en rapport avec la Loi sur la protection de la jeunesse}

Depuis 1979, les analystes de la protection de la jeunesse ont, pour la plupart, limité leurs études à l'action des centres de services sociaux. Si la Loi sur la protection de la jeunesse mandate les directeurs de la protection de la jeunesse à intervenir dans les situations où la sécurité et le développement de l'enfant sont compromis, la protection de la jeunesse, elle, commande des actions substantiellement plus larges.

Elle interpelle le réseau de la santé et des services sociaux, les organismes communautaires et la justice. Elle exige, de plus, une participation élargie de l'ensemble des citoyens sous forme de sensibilisation et de responsabilisation par rapport aux enfants en difficulté (les signalants). Mais sans l'assistance de puissants autres intervenants, la protection de la jeunesse ne sera rien d'autre qu'un baume sur des plaies vives.

Nous pensons ici à une action énergique au chapitre des déterminants du bien-être et de la santé. Sans une intervention concertée et énergique sur le revenu, l'employabilité, les habitudes de bien-être et de santé, sans l'application large d'une politique familiale, la distribution de la richesse et de la pauvreté continuera de s'effectuer de façon 
de plus en plus inéquitable. Et on continuera de penser que la personne est seule responsable de ses difficultés.

Ce cadre général de redistribution de l'aisance et des difficultés constitue le cadre de référence des travailleurs sociaux. La protection de la jeunesse ne peut être appréciée véritablement sans y faire référence.

\section{Notes}

${ }^{1}$ Pierre Racine, "La Loi sur la protection de la jeunesse : son impact sur les professionnels ", Intervention, $n^{\circ} 84$, novembre 1989, p. 10 et 12.

${ }^{2}$ Benoît Blanchet et Michel K. Laflamme, Commission d'enquête sur les services de santé et les services sociaux. Dossier thématique : Évolution de la pratique sociale au Québec de 1970 à 1986 dans les services Enfance-Famille, 12 décembre 1986, p. 107.

${ }^{3}$ Louise Boucher, Jean Gosselin et Danielle Guay, "Le concept de protection : se questionner pour transformer la pratique ", Intervention, $\mathrm{n}^{\circ} 72$, septembre 1985, p. 59.

${ }^{4}$ Encyclopedia of Social Work, 18th edition, 1987. Et notes personnelles de Gilles Lacroix, conseiller-cadre à la DSP du CSSCQ.

${ }^{5}$ Frédéric Lesemann et Gilbert Renaud, "Loi 24 et transformation des pratiques professionnelles en service social », Intervention, $n^{\circ} 58$, été 1980, p. 25 et 57 .

${ }^{6}$ Robert Dubé et Marjolaine St-Jules, Protection de l'enfance, réalité de l'intervention. Montréal, Gaëtan Morin Éditeur, 1987, p. 127 et ss.

${ }^{7}$ Benoît Blanchet et Pierre Pinard, Commission d'enquête sur les services de santé et les services sociaux, Le concept de protection, une comparaison Québec-Ontario, 1987.

${ }^{8}$ Jean Harvey, Rapport sur l'analyse des activités de réception et de traitement des signalements, et d'évaluation et d'orientation en protection de la jeunesse, 24 mars 1988.

${ }^{9}$ Jean Harvey, Protocoles, réception et traitement des signalements évaluation et orientation. Suites au rapport sur l'analyse des activités de réception et de traitement des signalements, et d'évaluation et d'orientation en protection de la jeunesse (Rapport Harvey), décembre 1988.

${ }^{10}$ Jean Harvey, Rapport sur l'analyse des activités de réception et de traitement des signalements, et d'évaluation et d'orientation en protection de la jeunesse. Annexes. Montréal, 24 mars 1988. Annexe 6, p. 1-5.

${ }^{11}$ Pierre Racine, op. cit.

${ }^{12}$ Hélène Manseau, L'abus sexuel et l'institutionnalisation de la protection de la jeunesse, Presses de l'Université du Québec, Québec, 1990.

${ }^{13}$ MSSS, Cadre de référence sur l'orientation et l'organisation des centres de réadaptation pour jeunes en difficulté d'adaptation, mai 1990. 